http://jmscr.igmpublication.org/home/

ISSN (e)-2347-176x ISSN (p) 2455-0450

crossref DOI: https://dx.doi.org/10.18535/jmscr/v8i4.19

Journal Of Medical Science And Clinical Research

IGM Publication

An Official Publication of IGM Publication

\title{
Study on Correlation between High Sensitivity C-Reactive Protein Levels and Left Ventricular Function in Patients with Acute Coronary Syndrome
}

\author{
Authors \\ Dr Aman Deep Goel ${ }^{1 *}$, Dr Bhupinder Singh ${ }^{2}$, Dr Tarsem Pal Singh ${ }^{3}$ \\ ${ }^{1}$ Junior Resident, Department of Medicine, Government Medical College, Amritsar-143001 \\ ${ }^{2}$ Associate Professor, Department of Medicine, Government Medical College, Amritsar-143001 \\ ${ }^{3}$ Professor, Department of Medicine, Government Medical College, Amritsar-143001 \\ *Corresponding Author \\ Dr Aman Deep Goel \\ Department of Medicine, Government Medical College, Amritsar-143001, India
}

\begin{abstract}
Introduction: Present work was aimed to study the correlation of hs-CRP with left ventricular function in patients of NSTEMI, STEMI and unstable angina. This study was carried out in 120 patients of acute coronary syndrome, presenting to Guru Nanak Dev Hospital attached to Government Medical College, Amritsar.

Material and Methods: Present work is a prospective observational study to determine correlation between plasma hs-CRP levels and outcome in patients with acute coronary syndrome. The data was collected from the patients and recorded in a prepared Case Report Form. Demographic details, medical history, information on exercise, diet, substance use, and hospitalization details was collected. After relevant history and thorough clinical examination, height, weight and BMI were measured. Hs-CRP was measured in patients and echocardiography was performed.

Results: In the present study, the data showcases the relationship between hs- CRP and various echocardiographic parameters in patients with acute coronary syndrome. Observations of the present study shows the correlation between the mean hs-CRP and ejection fraction of individuals of NSTEMI, STEMI and Unstable angina.

Conclusions: The data concludes that hs-CRP is more closely related to LV function as assessed by ejection fraction. As our data demonstrated a significant correlation between high hs-CRP and reduced EF, increased hs-CRP may be a marker of LV dysfunction.

Keywords: hs-CRP, Acute coronary syndrome, Unstable angina, STEMI, NSTEMI.
\end{abstract}

\section{Introduction}

Cardiovascular disease is worldwide most common cause of death. Coronary artery disease (CAD) prevalence has been increasing in rural India as well as in urban India. Acute myocardial infarction (AMI) is overwhelmingly the most important form of ischemic heart disease which continues to be the leading cause of death in the industrialized as well as developing countries like India, despite there being lot of progress in their prevention, detection and treatment over last 30 years. Hence, myocardial infarction remains an important health problem.

The acute manifestation of CAD is Acute Coronary Syndrome (ACS), subdivided into sudden cardiac death, Non ST elevation ACS (NSTEACS) and ST-elevation myocardial infarction (STEMI). The NSTEACS is further subdivided into Non ST-elevation myocardial infarction (NSTEMI) and Unstable angina (UA). 
ST elevation myocardial infarction (STEMI) is caused by acute occlusion of a major coronary artery usually due to disruption of an atherosclerotic plaque with subsequent formation of an occluding thrombus ${ }^{[1]}$. Effective and rapid restoration of blood flow to ischemic myocardial tissue is the most important initial goal in the treatment of patients with STEMI.

Certain primary risk factors have been identified with the development of atherosclerotic coronary artery disease and myocardial infarction: dyslipidemia, diabetes mellitus, hypertension, smoking, male gender, obesity and family history of atherosclerotic arterial disease. In presence of any risk factor, the relative risk of developing atherosclerotic coronary artery disease doubles. Many of the modifiable risk factors like physical activity, cessation of tobacco use, control of hypertension and diabetes mellitus are associated with lower risk profile and reduce the incidence of heart attack. Non-modifiable risk factors include age, gender and family history, which are considered to be reflecting a genetic predisposition.

Creatine Kinase-MB fraction is a cardiac marker used to assist diagnosis of an acute myocardial infarction. It measures the blood level of Creatine Kinase Myocardial Band (CK-MB). Its levels can be detected within 3 to 8 hours of the onset of chest pain, peak within 12 to 24 hours, and usually return to baseline levels within 24 to 48 hours.

C-reactive protein is a classical acute phase reactant, derived from liver, the serum level of which has long been known to increase after myocardial infarction ${ }^{[2]}$. C-reactive protein levels partially reflect the extent of myocardial necrosis. Elevated plasma C-reactive protein levels in patients with acute coronary syndromes on admission may indicate a state of persistent inflammation with poor short term prognosis ${ }^{[3]}$. Elevated C-reactive protein levels after AMI are associated with adverse clinical outcomes including cardiac rupture, left ventricular remodeling and sudden cardiac death. Increased CRP levels may portend the vulnerability of an atherosclerotic plaque and also contribute to plaque disruption ${ }^{[4]}$. It has a high specificity, positive predictive value and overall relative risk for prediction of an outcome ${ }^{[5]}$.

\section{Material and Methods}

Present work is a prospective observational study, conducted at Department of Medicine, Government Medical College, Amritsar. This study aims to determine correlation between plasma high sensitivity C-reactive protein (hsCRP) levels and outcome in patients with acute coronary syndrome. The subjects were consecutive patients who presented with a diagnosis of ACS, at the hospital. Once the patients met the inclusion and exclusion criteria as defined, they were enrolled in the study after signing the informed consent. The data was collected from the patients and recorded in a prepared Case Report Form Demographic details, medical history, information on exercise, diet, substance use, and hospitalization details was collected.

The diagnosis of ACS was made by all or any one of the following methods:

Chest pain/discomfort defined as any symptom of chest discomfort, sensation or pressure, or tightness; or arm, neck, or jaw pain occurring before hospital arrival or preceding a diagnosis of acute MI, NSTEMI or unstable angina. The chest pain/discomfort variable was classified as present or absent before admission, during admission, or both and was included (but was not limited to) in patients presenting with shortness of breath, nausea/vomiting, palpitations, syncope, or cardiac arrest.

All cases were divided into following groups:

1. STEMI patients were defined as having chest pain for at least 20 minute with the following electrocardiography changes: ST-segment elevation $>2 \mathrm{~mm}$ in 2 contiguous precordial leads or $>1 \mathrm{~mm}$ in 2 limb leads, new left bundle branch block, or electrocardiography changes compatible with true posterior MI, with elevation of cardiac enzyme levels above the reference range.

2. NSTEMI patient were defined as having 
elevation of cardiac markers (CK-MB or troponins) in the blood on admission or 6 hours of admission.

3. Unstable angina patients were defined as patients with ACS in whom there is no detectable release of the enzymes and biomarkers of myocardial necrosis (CKMB or troponins)

Time frame of Study- Data was collected for a period of 1 year and 4 months (from June 2018 to October 2019)

Inclusion Criteria- All patients included in the study satisfied the following criteria:

a) Patients with ACS.

b) Patients who were willing to sign an informed consent

\section{Exclusion Criteria -}

a. Patients with other conditions known to modify serum CRP such as:

○ Septicaemia

- Advanced liver disease

- Other inflammatory conditions such as chronic obstructive pulmonary disease, bronchitis.

Data Collection- The data collection was done on prepared Case records. The following information was collected from the patients who satisfy the inclusion criteria and were enrolled in the study.

- Demographic Details: The demographic details of the patient such as age (in years). Height (in centimeters), weight (in kilograms) and Body mass index were collected.

- Medical History: The occurrence of Diabetes Mellitus, Hypertension and Dyslipidemia were recorded.

- Medication History: Medication history of the patients was recorded.

- Lifestyle Factors: The level of exercise of the patient along with his/her diet and tobacco use were noted.

- Hospitalization details: The outcome of the hospital stay, treatment variables were recorded. The following parameters were collected;

A list of presenting complaints.
Details of hospital admission (in and out date)

$>$ List of the diagnostic tests performed before and during the hospital stay, such as ECG, 2D ECHO.

$>$ List of blood tests performed before and during the hospital visits per the practice (e.g.: Hemoglobin, total count, red blood cell, differential count, platelets, random blood sugar, serum creatinine, blood urea nitrogen (BUN), lipid profile, hs-CRP, troponin T).

Cardiac death was defined as a death due to myocardial infarction, cardiac arrhythmias (sustained ventricular tachycardia, ventricular fibrillation and supraventricular tachycardia with hemodynamic compromise), cardiogenic shock, thrombo-embolism or congestive cardiac failure.

Samples Collection- Venous blood samples were taken from all subjects on admission up to 6 hours after admission. $5 \mathrm{ml}$ of blood without anticoagulant was collected from each participant using vacutainer system tubes (Becton-Dickinson). Samples were centrifuged at $3000 \mathrm{rpm}$ for 15 minutes for rapid serum separation.

Laboratory Measurements- hs-CRP was determined by using BN2 nephelometer using the nephelometry technique. The detection limit was $0.1 \mathrm{mg} / \mathrm{L}$, the assay was linear from 0.16 to 255 $\mathrm{mg} / \mathrm{L}$. The 95 th percentile in 120 healthy donors in our institution was established at $3.0 \mathrm{mg} / \mathrm{L}$. Left ventricular function in all the patients was assessed by echocardiography with the help of GE and Philips EPIC-7 machines.

Data Collection and Statistical Analysis- Data was entered in the prepared Case Report Forms. Statistical analysis was performed after checking the data consistency. All the qualitative data was described as simple frequencies with percentage. Quantitative data was expressed as mean, range, and 95 percent confidence limits. Descriptive statistics is calculated for all variables. SPSS-22 version of software was used, released 2013, Armonk, NY: IBM Corp. 
- Difference in mean were tested using t-test or Mann-Whitney U test as appropriate.

- Differences in proportion were assessed using Chi-square or Fisher's exact test.

- For all tests, 2 sided p-value of 0.05 or less was considered significant.

\section{Observation}

Number of patients in 20-40 years age group were $2(5 \%)$ in NSTEMI, 2 (5\%) in STEMI and 6 (15 $\%)$ in Unstable angina. Number of patients in 4160 years age group were $24(60 \%)$ in NSTEMI, 25 (62.50) in STEMI and $24(60 \%)$ in Unstable angina. Number of patients in 61-80 years age group were 14 (35\%) in NSTEMI, 13 (32.50\%) in STEMI and 10(25\%) in Unstable angina. The mean age of patients was 58.28 years in NSTEMI, 59.13 years in STEMI and 56.98 yrs in Unstable angina which was found to be statistically non significant between the groups (Table 1).

Table 1 Distribution of age group in patients of NSTEMI, STEMI and Unstable Angina

\begin{tabular}{|l|c|c|c|c|c|c|c|c|}
\hline \multirow{2}{*}{$\begin{array}{l}\text { Age } \\
\text { group }\end{array}$} & \multicolumn{9}{|c|}{ Diagnosis } & \multicolumn{2}{c|}{ Total } \\
\cline { 2 - 8 } & NSTEMI & \multicolumn{2}{|c|}{ STEMI } & \multicolumn{2}{c|}{$\begin{array}{c}\text { UNSTABLE } \\
\text { ANGINA }\end{array}$} & No. & $\%$ \\
\cline { 2 - 8 } & No. & $\%$ & No. & $\%$ & No. & $\%$ & & \\
\hline $20-40$ & 2 & 5.00 & 2 & 5.00 & 6 & 15.00 & 10 & 8.33 \\
\hline $41-60$ & 24 & 60.00 & 25 & 62.50 & 24 & 60.00 & 73 & 60.83 \\
\hline $61-80$ & 14 & 35.00 & 13 & 32.50 & 10 & 25.00 & 37 & 30.84 \\
\hline Total & 40 & 100.0 & 40 & 100.0 & 40 & 100.00 & 120 & 100.0 \\
\hline Mean & $58.28 \pm 10.78$ & $59.13 \pm 9.43$ & $53.55 \pm 12.40$ & $56.98 \pm 11.12$ \\
\hline $\begin{array}{l}\text { p- } \\
\text { value }\end{array}$ & \multicolumn{8}{|c|}{0.053} \\
\hline
\end{tabular}

Table 2 shows distribution of individuals according to sex. Out of total 40 patients in each group, males were 20 (50\%), 21 (52.50) and 18 (45 \%) in NSTEMI, STEMI and unstable angina respectively and females were 20 (50\%), 19 $(47.50 \%)$ and $22(55 \%)$ in NSTEMI, STEMI and unstable angina respectively.

Table 2 Distribution of the patients across the groups depending on sex

\begin{tabular}{|l|c|c|c|c|c|c|c|c|}
\hline Sex & \multicolumn{6}{|c|}{ Diagnosis } & \multicolumn{2}{c|}{ Total } \\
\cline { 2 - 8 } & \multicolumn{2}{|c|}{ NSTEMI } & \multicolumn{2}{|c|}{ STEMI } & \multicolumn{2}{c|}{$\begin{array}{c}\text { UNSTABLE } \\
\text { ANGINA }\end{array}$} & No. & $\%$ \\
\cline { 2 - 7 } & No. & $\%$ & No. & $\%$ & No. & $\%$ & & \\
\hline Female & 20 & 50.00 & 19 & 47.50 & 22 & 55.00 & 61 & 50.83 \\
\hline Male & 20 & 50.00 & 21 & 52.50 & 18 & 45.00 & 59 & 49.17 \\
\hline Total & 40 & 100.00 & 40 & 100.0 & 40 & 100.00 & 120 & 100.00 \\
\hline
\end{tabular}

Table 3 shows the distribution of individuals of NSTEMI, STEMI and Unstable angina according to their outcome. The number of patients who survived was $35(87.50 \%)$ in NSTEMI, 38 (95\%) in STEMI and $33(82.50 \%)$ in Unstable angina. The number of patients who expired was 5 $(12.50 \%)$ in NSTEMI, $2(5 \%)$ in STEMI and 6 $(15 \%)$ in Unstable angina. Out of all patients taken for study, 1 (2.50\%) patient of unstable angina went LAMA.

Table 3 Distribution of the patients across the groups depending outcome

\begin{tabular}{|c|c|c|c|c|c|c|c|c|}
\hline \multirow[t]{3}{*}{ Outcome } & \multicolumn{6}{|c|}{ Diagnosis } & \multicolumn{2}{|c|}{ Total } \\
\hline & \multicolumn{2}{|c|}{ NSTEMI } & \multicolumn{2}{|c|}{ STEMI } & \multicolumn{2}{|c|}{$\begin{array}{l}\text { UNSTABLE } \\
\text { ANGINA }\end{array}$} & \multirow[t]{2}{*}{ No. } & \multirow[t]{2}{*}{$\%$} \\
\hline & No. & $\%$ & No. & $\%$ & No. & $\%$ & & \\
\hline ALIVE & 35 & 87.50 & 38 & 95.00 & 33 & 82.50 & 106 & 88.33 \\
\hline EXPIRED & 5 & 12.50 & 2 & 5.00 & 6 & 15.00 & 13 & 10.83 \\
\hline LAMA & 0 & 0.00 & 0 & 0.00 & 1 & 2.50 & 1 & 0.83 \\
\hline Total & 40 & 100.0 & 40 & 100.0 & 40 & 100.0 & 120 & 100.0 \\
\hline
\end{tabular}

Table 4 shows the average systolic blood pressure of individuals of NSTEMI, STEMI and Unstable angina. The average systolic blood pressure was $137.65 \pm 18.81 \mathrm{mmHg}$ in NSTEMI, 136.60 \pm 24.91 in STEMI and $113.45 \pm 16.31$ in Unstable angina.

Table 4 Systolic blood pressure

\begin{tabular}{|l|c|c|c|}
\hline $\begin{array}{l}\text { Systolic } \\
\text { Blood } \\
\text { Pressure }\end{array}$ & NSTEMI & STEMI & $\begin{array}{c}\text { UNSTABLE } \\
\text { ANGINA }\end{array}$ \\
\cline { 2 - 4 } & & $136.60 \pm 24.91$ & $113.45 \pm 16.31$ \\
\hline Mean & $137.65 \pm 18.81$ & 0.001 \\
\hline p-value & \multicolumn{3}{|c|}{ Diagnosis } \\
\hline
\end{tabular}

Table 5 shows the average diastolic blood pressure of individuals of NSTEMI, STEMI and Unstable angina. The average diastolic blood pressure was $91.25 \pm 15.03 \mathrm{mmHg}$ in NSTEMI, 86.75 $\pm 15.03 \mathrm{in}$ STEMI and $74.85 \pm 12.45$ in Unstable angina.

Table 5 Diastolic blood pressure

\begin{tabular}{|c|c|c|c|}
\hline \multicolumn{4}{|c|}{\begin{tabular}{|l|l} 
Diastolic & Diagnosis
\end{tabular}} \\
\hline $\begin{array}{l}\text { blood } \\
\text { pressure }\end{array}$ & NSTEMI & STEMI & $\begin{array}{l}\text { UNSTABLE } \\
\text { ANGINA }\end{array}$ \\
\hline Mean & $91.25 \pm 15.03$ & $86.75 \pm 15.03$ & $74.85 \pm 12.45$ \\
\hline p-value & & 0.001 & \\
\hline
\end{tabular}

Table 6 and Figure 1. Showing the correlation between the mean hs-CRP and ejection fraction of individuals of NSTEMI, STEMI and Unstable angina. 
Table 6.

\begin{tabular}{|l|c|c|c|c|}
\hline \multirow{2}{*}{} & \multicolumn{4}{|c|}{ Diagnosis } \\
\cline { 2 - 5 } & NSTEMI & STEMI & $\begin{array}{c}\text { UNSTABLE } \\
\text { ANGINA }\end{array}$ & p- value \\
\hline hs-CRP & $42.19 \pm 59.26$ & $47.63 \pm 56.84$ & $5.22 \pm 8.53$ & 0.001 \\
\hline $\begin{array}{l}\text { Ejection } \\
\text { fraction }\end{array}$ & $49.00 \pm 9.43$ & $45.05 \pm 9.36$ & $54.08 \pm 10.94$ & 0.002 \\
\hline
\end{tabular}

\section{Figure 1}

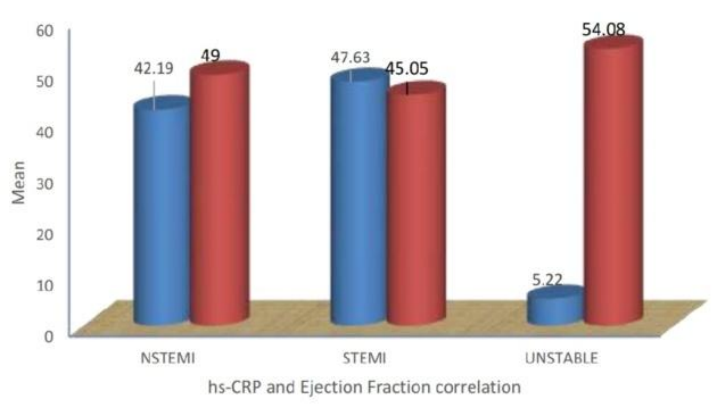

\section{Discussion}

The present study showed that the acute phase reactant, hs-CRP, is significantly elevated in patients with acute coronary syndromes. The number of patients with CRP elevations was much higher in NSTEMI and STEMI (80\% and 72.5\%) compared to unstable angina (40\%). The hs-CRP levels were much more significantly high in NSTEMI and STEMI compared to Unstable Angina. The mean CRP levels in NSTEMI and STEMI were $42.19 \pm 59.26 \mathrm{mg} / \mathrm{L}$ with a range of 0.16 to $255 \mathrm{mg} / \mathrm{L}$ and $47.63 \pm 56.84 \mathrm{mg} / \mathrm{L}$ with a range of 0.56 to $184 \mathrm{mg} / \mathrm{L}$ respectively as compared to Unstable Angina where the mean hsCRP was $5.22 \pm 8.53 \mathrm{mg} / \mathrm{L}$ with a range of 0.16 to $40.7 \mathrm{mg} / \mathrm{L}$. It was seen from the study that the hsCRP levels were more elevated in patients with STEMI in comparison to NSTEMI, signifying a greater degree of inflammation and probably myocardial damage as well. A study by Mach et al ${ }^{[8]}$ showed that among patients with acute ischemic heart disease and no biological markers of myocardial necrosis, the CRP concentration at the time of admission was significantly higher in patients in whom an acute myocardial infarction was ultimately diagnosed, while in patients with unstable angina the CRP levels were low.

The present study results are comparable to the study by Sheikh et al ${ }^{[7]}$ who also demonstrated higher number of patients with elevated CRP and higher mean levels of CRP in NSTEMI and STEMI compared to Unstable Angina and control group. The present study results are also comparable to the study by Cavusoglu et al ${ }^{[9]}$ who demonstrated that the CRP concentrations in patients presenting with acute coronary syndromes, within 6 hours of onset of symptoms were significantly higher as compared to the Control Group. The inflammatory process has been shown to be one of the mechanisms causing plaque rupture leading to elevated CRP levels in less than 6 hours in patients with acute coronary syndrome ${ }^{[10]}$. In patients presenting with ACS, hsCRP concentrations were more than 10-fold higher than in patients with stable coronary disease or no known coronary disease.

In the present study, we correlated the mean hsCRP and left ventricular ejection fraction in individuals of NSTEMI, STEMI and Unstable angina which concluded that in patients with high values of hs-CRP levels, LV ejection fraction was lower. According to Silva and Pais de Lacerda ${ }^{[6]}$, hs- CRP concentration has a direct correlation with coronary artery remodeling grade, atherosclerotic plaque content (assessed using intravascular ultrasound) and an indirect correlation with collateral coronary circulation, left ventricle ejection fraction.

In the present study mortality occurred in 5\% $(\mathrm{n}=2), 12.5 \%(\mathrm{n}=5)$ and $15 \%(\mathrm{n}=6)$ patients of STEMI, NSTEMI and Unstable Angina groups respectively. In patients with high hs-CRP (hsCRP >3) mortality occurred in $16.9 \% \quad(n=13)$ patients as compared to $0 \%(n=0)$ patients with low hs-CRP. In prior studies, increased concentrations of hs-CRP have been shown to be associated with mortality in patients with UA and NSTEMI ${ }^{[11]}$.

In 2003 American Centers of Disease Control and Prevention (CDC) as well as American Heart Association (AHA) have recommended implementing hs-CRP as an independent prognostic factor in patients with ACS. It is estimated that a CRP value of greater than 10 $\mathrm{mg} / \mathrm{L}$ prognosticates the risk of ACS recurrence. The prognostic value of hs-CRP does not depend 
on troponin concentration, hence can be a useful risk evaluation tool for those without signs of myocardial necrosis.

According to the meta-analysis by He et al ${ }^{[12]}$ (20 studies, 17,442 patients), early rise of hs-CRP (within $72 \mathrm{~h}$ from the start of ACS) is moderately associated with long term cardiovascular event recurrence or death risk. In ACS sufferers, hs-CRP concentration of $3.1-10.0 \mathrm{mg} / \mathrm{L}$ is related to 1.4 , and $>10 \mathrm{mg} / \mathrm{L}$ is related to 2.18 times higher adverse event risk. Moreover, higher hs-CRP concentration is related to greater myocardial damage, and the intensity of early inflammatory response is related to ventricular function and remodelling, ischemic and reperfusion damage, all of which can be significant for long-term outcomes. The drawbacks of meta-analysis are as follows: insufficient number of proper studies, different values of hs-CRP (expressed by logarithmic or categorical values), not all the studies evaluated the influence of significant risk factors (drugs, damage extent), articles analysed were only in English. The 2011 ESC guidelines on non-ST-elevation ACS draw attention that the hsCRP concentration of $>0.1 \mathrm{mg} / \mathrm{L}$ in patients with normal troponin concentration is related to longterm (from 6 months to 4 years) mortality. In order to estimate moderate and long-term risk, hs-CRP estimation can be done after the episode of ACS.

The present data suggests that the intensity of the inflammatory response increases the risk of mechanical consequences and complications of ischemic injury and therefore may play a role in the development of heart failure. Increased concentrations of CRP may then help to identify patients at risk of developing congestive heart failure after ACS and prompt closer surveillance and more aggressive therapy and perhaps novel therapy aimed at prevention of adverse remodeling. Assessment of CRP on admission provides independent prognostic information and thereby improves the ability to identify those patients at highest risk of death and heart failure. Liuzzo et al ${ }^{[13]}$ demonstrated that CRP elevation in patients with unstable angina, without evidence of myocardial damage as assessed with troponin $\mathrm{T}$, is associated with poor outcome. In another study, Liuzzo et al ${ }^{[14]}$ showed that severe myocardial ischemia in patients with variant angina without atherosclerotic coronary artery disease does not by itself induce an increase in plasma CRP. Therefore, it is likely that CRP elevations are due to activation of inflammation. It has been shown that other pro-inflammatory cytokines such as interleukin-6, interleukin-8 and TNF-a are elevated on admission in patients with acute coronary syndromes and that these elevations may be associated with a worse outcome ${ }^{[15]}$.

In the present study, the mean ejection fraction of individuals of NSTEMI, STEMI and Unstable angina was $49.00 \pm 9.43 \mathrm{mmHg}$ in NSTEMI, $45.05 \pm 9.36$ in STEMI and 54.08 \pm 10.94 in Unstable angina. The study presents data regarding the relationship between hs-CRP and various echocardiographic parameters in patients with acute coronary syndrome. The data led us to the conclusion that hs-CRP is more closely related to $\mathrm{LV}$ function as assessed by ejection fraction. As our data demonstrated a significant correlation between high hs-CRP and reduced EF, increased hs- CRP may be a marker of LV dysfunction.

\section{Bibliography}

1. Libby P. Current Concepts of the Pathogenesis of the Acute Coronary Syndromes. Circulation. 2001;104(3):36572.

2. Ross R. Atherosclerosis-an inflammatory disease. N Engl J Med. 1999;340(2):11526.

3. Calabrò P, Golia E, Yeh ET. CRP and the risk of atherosclerotic events. Semin Immunopathol 2009;31(1):79-94.

4. Blake GJ, Ridker PM. Novel clinical markers of vascular wall inflammation. Circ Res 2001; 89:763-71

5. Calabro P, Willerson JT, Yeh ET. Inflammatory cytokines stimulated Creactive protein production by human coronary artery smooth muscle cells. Circulation. 2003;108(16):1930-32.

6. Silva D, Pais de Lacerda A. High- 
sensitivity C-reactive protein as a biomarker of risk in coronary artery disease. Rev Port Cardiol 2012; 31(11):733-45.

7. Sheikh AS, Yahya S, Sheikh NS, Sheikh AA. C-reactive protein as a predictor of adverse outcome in patients with acute coronary syndrome. Heart Views. 2012;13:7-12.

8. Mach F, Lovies C, Gaspoz JM, Unger PF, Bouilli $\mathrm{M}$, Urban $\mathrm{P}$ et al. C- reactive protein as a marker for acute coronary syndromes. Eur Heart J. 1997;18:897-902.

9. Cavusoglu Y, Gorenek B, Alpsoy S, Unalir A, Ata N, Timuralp B. Evaluation of Creactive protein, fibrinogen and antithrombin III as risk factors for coronary artery disease. Isr Med Assoc J. 2001;3:368.

10. Tomoda H, Aoki N. Prognostic value of Creactive protein levels within six hours after the onset of acute myocardial infarction. Am Heart J. 2000;140:324-8.

11. Beck B, Weintraub WS, Alexander R. Elevation of C-reactive protein in "active" coronary artery disease. Am J Cardiol. 1990;65:168-72.

12. He L, Tang X, Ling W, Chen W, Chen Y. Early C-reactiveprotein in the prediction of long-term outcomes after acutecoronary syndromes: a meta-analysis of longitudinal studies. Heart. 2010;96:339-46.

13. Liuzzo G, Biasucci LM, Gallimore JR, Grillo RL, Rebuzzi AG, Pepys MB et al. The prognostic value of $\mathrm{C}$-reactive protein and serum amyloid $\mathrm{A}$ protein in severe unstable angina. $\mathrm{N}$ Engl J Med. 1994;331:417-24.
14. Liuzzo G, Biasucci LM, Rebuzzi AG, Gallimore JR, Caligiuri G, Lanza GA et al. Plasma protein acute phase response in unstable angina is not induced by ischemic injury. Circulation. 1996;94:2372-80

15. Biasucci LM, Vitelli A, Liuzzo G, Altamura S, Caligiuri G, Monaco $\mathrm{C}$ et al. Elevated levels of interleukin-6 in unstable angina. Circulation. 1996; 94:874. 